\title{
Particle Production via Dirac Dipole Moments in the Magnetized and Nonmagnetized Exponentially Expanding Universe
}

\author{
Semra Gurtas Dogan, ${ }^{1}$ Ganim Gecim, ${ }^{2}$ and Yusuf Sucu ${ }^{1}{ }^{1}$ \\ ${ }^{1}$ Department of Physics, Faculty of Science, Akdeniz University, 07058 Antalya, Turkey \\ ${ }^{2}$ Department of Astronomy and Astrophysics, Faculty of Science, Atatürk Univ., 25240 Erzurum, Turkey \\ Correspondence should be addressed to Yusuf Sucu; ysucu@akdeniz.edu.tr
}

Received 5 April 2019; Revised 25 June 2019; Accepted 7 July 2019; Published 22 July 2019

Academic Editor: Kazuharu Bamba

Copyright (C) 2019 Semra Gurtas Dogan et al. This is an open access article distributed under the Creative Commons Attribution License, which permits unrestricted use, distribution, and reproduction in any medium, provided the original work is properly cited. The publication of this article was funded by SCOAP ${ }^{3}$.

\begin{abstract}
In the present paper, we solve the Dirac equation in the $2+1$ dimensional exponentially expanding magnetized by uniform magnetic field and nonmagnetized universes, separately. Asymptotic behaviors of the solutions are determined. Using these results we discuss the current of a Dirac particle to discuss the polarization densities and the magnetization density in the context of Gordon decomposition method. In this work we also calculate the total polarization and magnetization, to investigate how the magnetic field affects the particle production. Furthermore, the electric and the magnetic dipole moments are calculated, and based on these, we have discussed the effects of the dipole moments on the charge distribution of the universe and its conductivity for both the early and the future time epoch in the presence/absence of a constant magnetic field and exponentially expanding spacetime.
\end{abstract}

\section{Introduction}

One of the most interesting and important results of the formulation of the relativistic quantum mechanics in curved spacetime is the particle creation event in the expanding universe which was firstly discussed by Parker for the scalar particles and Dirac particles [1-3]. So, he computed the number density of the created particles by means of the Bogolibov transformation by using the out vacuum states constructed from the solutions of the relativistic particles wave equations. After these important works of Parker, the solutions of the relativistic particle wave equations have extensively been studied in various $3+1$ dimensional spacetime backgrounds [4-19]. Using the WKB approach, the number density and renormalized energy-momentum tensor of the created spin$1 / 2$ particle in the spatially flat (3+1)-dimensional FriedmannRobertson-Walker (FRW) spacetime have been calculated [20]. The effect of the scalar particle creation on the collapse of a spherically symmetric massive star was investigated and it was demonstrated that the collapsing process was not independent from the particle creation rate [21]. Moreover, in [22] thermodynamics laws and equilibrium conditions are discussed in the presence of particle creation in the context of the $(3+1)$-dimensional Chern-Simons gravity theory. Recently, creation of the massless fermion in the Bianchi type-I spacetime investigated and showed that the massless particles can be created during the early anisotropic expansion epoch [23].

The Gordon decomposition of the Dirac currents is another useful tool for discussing the particle creation phenomena $[4,9,10,24]$. In the decomposition method, the Dirac currents constructed from the solutions of the Dirac equations are separated into three parts, the convective, polarization with three components, and magnetization with three components, in the $3+1$ dimensional spacetime. This method includes some complexities stemming from the $3+1$ dimensional spacetime. Using this method in a $2+1$ dimensional curved spacetime, the densities of the particle currents are separated into three parts, as in the $3+1$ dimensional spacetime, but polarization density has two components and the magnetization density has only one component [24], and, moreover, as the Dirac spinor can be defined by only two components, the computations in 
the $2+1$ dimensional spacetime become more simple than that of $3+1$ dimensional spacetime. Because of the simplicity stemming from the dimensions, the dipole moments that are computed from the polarization and magnetization densities of the Dirac electron under influence in a constant magnetic field are easily computed and their result are, furthermore, compatible with the current experimental results [25]. With these motivations, in this study, we solve the Dirac equation in the $2+1$ dimensional exponentially expanding magnetized by uniform magnetic field and nonmagnetized universes, separately, and discuss the particle creation event by means of the Dirac currents written in terms of these solutions. As a result, we observe that the polarization and magnetization parts of the currents are affected differently whether the exponentially expanding universe is magnetized or not and find expressions for the electric and magnetic dipole moments by integrating the polarization and magnetization densities on hypersurface.

The outline of the work is as follows; in Section 2, we, at first, discuss the Dirac equation solutions in the $2+1$ dimensional exponentially expanding universe. In Section 3, the Dirac equation is solved in the $2+1$ dimensional exponentially expanding universe with a constant magnetic field. In Section 4, we derive the components of Dirac currents for the solutions obtained in Sections 2 and 3 and also compute the polarization density, the magnetization density, total polarization (electric dipole moment), and the total magnetization (magnetic dipole moment). Finally, the last section, conclusion, includes a discussion about the results of this work.

\section{Dirac Particle in the $2+1$ Dimensional Exponentially Expanding Universe}

The behavior of the electron in $2+1$ dimensional curved space is represented by the covariant form of the Dirac equation [24], which is important application in curved spacetime [2633]

$$
\left\{i \bar{\sigma}^{v}(x)\left[\partial_{\nu}-\Gamma_{v}+i e A_{\nu}\right]\right\} \Psi(x)=m \Psi(x),
$$

where $\Psi(x)=\left(\begin{array}{l}\Psi_{1} \\ \Psi_{2}\end{array}\right)$ is the Dirac spinorial wave function with two components that are positive and negative energy eigenstates, $m$ is the mass of Dirac particle, $e$ is the charge of the Dirac particle, and $A_{v}$ are 3-vectors of electromagnetic potential. Using triads, $e_{(i)}^{v}(x)$, Dirac matrices that dependent on spacetime, $\bar{\sigma}^{v}(x)$, are written in terms of the constant Dirac matrices, $\bar{\sigma}^{i}$;

$$
\bar{\sigma}^{v}(x)=e_{(i)}^{v}(x) \bar{\sigma}^{i}
$$

So, we choose the constant Dirac matrices, $\bar{\sigma}^{i}$, in the flat spacetime as follows:

$$
\bar{\sigma}^{i}=\left(\bar{\sigma}^{0}, \bar{\sigma}^{1}, \bar{\sigma}^{2}\right)
$$

with

$$
\begin{aligned}
& \bar{\sigma}^{0}=\sigma^{3}, \\
& \bar{\sigma}^{1}=i \sigma^{1}, \\
& \bar{\sigma}^{2}=i \sigma^{2},
\end{aligned}
$$

where $\sigma^{1}, \sigma^{2}$, and $\sigma^{3}$ are Pauli matrices. The spin connection, $\Gamma_{\nu}(x)$, for the diagonal metrics is defined as

$$
\Gamma_{\nu}(x)=-\frac{1}{4} g_{\tau \alpha} \Gamma_{\nu \beta}^{\alpha}\left[\bar{\sigma}^{\tau}(x), \bar{\sigma}^{\beta}(x)\right],
$$

where $\Gamma_{\nu \beta}^{\alpha}$ is the Christoffel symbol given as follows [34]:

$$
\Gamma_{\nu \beta}^{\alpha}=\frac{1}{2} g^{\lambda \alpha}\left[\partial g_{\beta \lambda, \nu}+\partial g_{\nu \lambda, \beta}-\partial g_{\beta \nu, \lambda}\right]
$$

Also, the metric tensor $g_{\beta \nu}(x)$ is written in terms of triads as follows:

$$
g_{\beta \nu}(x)=e_{\beta}^{(i)}(x) e_{\nu}^{(j)}(x) \eta_{(i)(j)},
$$

where $\beta$ and $\nu$ are curved spacetime indices run from 0 to 2 , $i$ and $j$ are flat spacetime indices run 0 to 2 , and $\eta_{(i)(j)}$ is the signature with $(1,-1,-1)$.

The $(2+1)$ dimensional de Sitter spacetime metric can be written as [35]

$$
d s^{2}=d t^{2}-e^{2 H t}\left[d r^{2}+r^{2} d \phi^{2}\right]
$$

where $H$ is Hubble parameter. From (3)-(8), the spin connections for the metric read

$$
\begin{aligned}
& \Gamma_{0}=0, \\
& \Gamma_{1}=-\frac{H}{2} e^{H t} \bar{\sigma}^{0} \bar{\sigma}^{1}, \\
& \Gamma_{2}=-\frac{1}{2}\left[r H e^{H t} \bar{\sigma}^{0} \bar{\sigma}^{2}+\bar{\sigma}^{1} \bar{\sigma}^{2}\right] .
\end{aligned}
$$

Using (2), (8), and (9), then the Dirac equation in the $2+1$ dimensional exponentially expanding universe becomes

$$
\begin{aligned}
& \left\{\bar{\sigma}^{0}\left(\partial_{t}+H\right)+i m+e^{-H t}\left[\bar{\sigma}^{1}\left(\partial_{r}+\frac{1}{2 r}\right)+\frac{\bar{\sigma}^{2}}{r} \partial_{\phi}\right]\right\} \\
& . \Psi(x)=0 .
\end{aligned}
$$

Letting (4) and (10) and the Dirac spinor, $\Psi(x)=\left(\begin{array}{l}\Psi_{1} \\ \Psi_{2}\end{array}\right)$, we write the Dirac equation in explicit form as follows:

$$
\begin{aligned}
& {\left[\partial_{t}+i m+H\right] \psi_{1}+i e^{-H t}\left[\partial_{r}+\frac{1}{2 r}-\frac{i}{r} \partial_{\phi}\right] \psi_{2}=0} \\
& {\left[\partial_{t}-i m+H\right] \psi_{2}-i e^{-H t}\left[\partial_{r}+\frac{1}{2 r}+\frac{i}{r} \partial_{\phi}\right] \psi_{1}=0 .}
\end{aligned}
$$

To find the solutions of (11), thanks to the separation of variables method, the wave function components can be defined as

$$
\left(\begin{array}{c}
\Psi_{1} \\
\Psi_{2}
\end{array}\right)=e^{i k \phi}\left(\begin{array}{c}
T_{1}(t) R_{1}(r) \\
T_{2}(t) R_{2}(r)
\end{array}\right) .
$$

By these definitions, the Dirac equation is separated into the following two differential equation systems:

$$
\begin{aligned}
& {\left[\frac{d}{d r}+\frac{1}{2 r}+\frac{k}{r}\right] R_{2}(r)=-\lambda R_{1}(r)} \\
& {\left[\frac{d}{d r}+\frac{1}{2 r}-\frac{k}{r}\right] R_{1}(r)=\lambda R_{2}(r)}
\end{aligned}
$$


and

$$
\begin{aligned}
& {\left[\frac{d}{d t}+i m+H\right] T_{1}(t)=i \lambda e^{-H t} T_{2}(t)} \\
& {\left[\frac{d}{d t}-i m+H\right] T_{2}(t)=i \lambda e^{-H t} T_{1}(t),}
\end{aligned}
$$

where $\lambda$ is a separation constant, and we find the solutions of (13) in terms of the Bessel and confluent hypergeometric functions as follows:

$$
\begin{aligned}
R_{1}(r) & =A J_{k-1 / 2}(\lambda r) \\
& =A \frac{e^{-i \lambda r}(\lambda r / 2)^{k-1 / 2}}{\Gamma(k+1 / 2)}{ }_{1} F_{1}[k, 2 k ; i 2 r \lambda], \\
R_{2}(r) & =B J_{k+1 / 2}(\lambda r) \\
& =B \frac{e^{-i \lambda r}(\lambda r / 2)^{k+1 / 2}}{\Gamma(k+3 / 2)}{ }_{1} F_{1}[k+1,2 k+2 ; i 2 r \lambda] .
\end{aligned}
$$

On the other hand, to solve (14), we must define a new variable such as $z=(\lambda / H) e^{-H t}$. With the definition $n=$ $-i(m / H)$, the solutions of (14) are obtained in terms of Bessel functions or confluent hypergeometric functions:

$$
\begin{aligned}
T_{1}(z) & =C z^{3 / 2} J_{n+1 / 2}(z) \\
& =C z^{3 / 2} \frac{e^{-i z}(z / 2)^{n+1 / 2}}{\Gamma(n+3 / 2)}{ }_{1} F_{1}[n+1,2 n+2 ; i 2 z] \\
T_{2}(z) & =D z^{3 / 2} J_{n-1 / 2}(z) \\
& =D z^{3 / 2} \frac{e^{-i z}(z / 2)^{n-1 / 2}}{\Gamma(n+1 / 2)}{ }_{1} F_{1}[n, 2 n ; i 2 z]
\end{aligned}
$$

Then, the wave function, $\Psi(x)$, can be written as

$$
\Psi=N z^{3 / 2} e^{i k \phi}\left(\begin{array}{l}
J_{n+1 / 2}(z) J_{k-1 / 2}(\lambda r) \\
J_{n-1 / 2}(z) J_{k+1 / 2}(\lambda r)
\end{array}\right)
$$

where $N$ is normalization constant. To find the normalization constant, we use the Dirac-delta normalization condition [4, 36, 37]:

$$
\int \sqrt{|g|} \mid \bar{\Psi} \bar{\sigma}^{0} \Psi d \sigma=\delta\left(\lambda-\lambda^{\prime}\right)
$$

where $g$ is determinant of the metric tensor and $\bar{\Psi}=\Psi^{\dagger} \bar{\sigma}^{0}$ and $d \sigma=d r d \phi$ for the surface $t=$ constant [25]. Thus, the normalization constant is computed as

$$
|N|^{2}=\frac{H^{2}}{4 \pi z \lambda J_{n-1 / 2}(z) J_{n+1 / 2}(z)},
$$

where we use the following relation $[4,36,37]$ :

$$
\int_{0}^{\infty} r J_{k+1 / 2}(\lambda r) J_{k+1 / 2}\left(\lambda^{\prime} r\right) d r=\frac{1}{\lambda} \delta\left(\lambda^{\prime}-\lambda\right) .
$$

\section{Dirac Particle in the $2+1$ Dimensional Exponentially Expanding Magnetized Universe}

It is interesting in discussing if the universe is under influence in an external constant magnetic field in the beginning time. Therefore, an electromagnetic potential can be chosen as $A_{0}=0$ and $\vec{A}(r, \phi)=(1 / 2) B_{0} r \hat{\phi}$ for a constant magnetic field in $2+1$ dimensional spacetime. Then, the Dirac equation in the $2+1$ dimensional exponential expanding universe with a constant magnetic field becomes

$$
\begin{aligned}
& \left\{\bar{\sigma}^{0}\left(\partial_{t}+H\right)+i m\right. \\
& \left.\quad+e^{-H t}\left[\bar{\sigma}^{1}\left(\partial_{r}+\frac{1}{2 r}\right)+\bar{\sigma}^{2}\left(\frac{1}{2 r} \partial_{\phi}+\frac{i e B_{0}}{2}\right)\right]\right\} \Psi(x) \\
& \quad=0
\end{aligned}
$$

and, thus, the explicit form of the equation is written as

$$
\begin{aligned}
& \left(\partial_{t}+i m+H\right) \psi_{1}+i e^{-H t}\left(\partial_{r}+\frac{1}{2 r}-\frac{i}{r} \partial_{\phi}+\frac{e B_{0}}{2}\right) \psi_{2} \\
& \quad=0 \\
& \left(\partial_{t}-i m+H\right) \psi_{2}-i e^{-H t}\left(\partial_{r}+\frac{1}{2 r}+\frac{i}{r} \partial_{\phi}-\frac{e B_{0}}{2}\right) \psi_{1} \\
& \quad=0
\end{aligned}
$$

To solve (22), we use the same procedure as the section before. The solutions of the equations are

$$
\Psi=\frac{N z^{3 / 2} e^{i k \phi}}{\sqrt{\rho}}\left(J_{n-1 / 2}(z)\left[\frac{(\rho-2 \eta-1)\left(\sqrt{e^{2} B_{0}^{2}-4 \lambda^{2}}-e B_{0}\right)}{2 \lambda \rho} W_{\kappa, \eta}(\rho)-\frac{\sqrt{e^{2} B_{0}^{2}-4 \lambda^{2}}}{\lambda \rho} W_{\kappa+1, \eta}(\rho)\right]\right)
$$

where $\kappa=-e k B_{0} / \sqrt{e^{2} B_{0}^{2}-4 \lambda^{2}}, \eta=k-1 / 2, \rho=$ $\sqrt{e^{2} B_{0}^{2}-4 \lambda^{2}} r$, and $N$ is normalization constant. As all the contributions for particle creation and dipole moments are taking place from the boundaries, we can write the wave function in the following asymptotic form [36]: 


$$
\Psi \sim N z^{3 / 2} e^{i k \phi} \rho^{\kappa-1 / 2} e^{-\rho / 2}\left(\begin{array}{c}
\frac{1}{\Gamma(n+3 / 2)}\left(\frac{z}{2}\right)^{n+1 / 2} \\
\frac{1}{\Gamma(n+1 / 2)}\left(\frac{z}{2}\right)^{n-1 / 2} \frac{\left[(\rho-2 \eta-1)\left(\sqrt{e^{2} B_{0}^{2}-4 \lambda^{2}}-e B_{0}\right)-2 \rho \sqrt{e^{2} B_{0}^{2}-4 \lambda^{2}}-e B_{0}\right]}{2 \lambda \rho}
\end{array}\right) .
$$

Then, the normalization constant can be obtained from (18) as follows:

$$
\begin{aligned}
&|N|^{2} \sim \frac{H^{2}(\eta+1 / 2)^{2}\left(1-4 n^{2}\right)}{\cos (n \pi)} \\
& \cdot \frac{1}{\left[z^{2}\left((\eta+1 / 2)^{2}-\kappa^{2}\right) \Gamma(2 \kappa)+\left(1-4 n^{2}\right) \Gamma(2 \kappa-2)\right]},
\end{aligned}
$$

where we use $\Gamma(-n+3 / 2) \Gamma(n+3 / 2)=\pi\left(1-4 n^{2}\right) / 4 \cos (n \pi)$ and $\Gamma(-n+1 / 2) \Gamma(n+1 / 2)=\pi / \cos (n \pi)$.

\section{Dirac Currents}

The $2+1$ dimensional Dirac current is written as

$$
J^{v}=\bar{\Psi} \bar{\sigma}^{v}(x) \Psi
$$

where $\bar{\Psi}$ is Hermitian conjugate of the Dirac spinor $\Psi$ and equal to $\bar{\Psi}=\Psi^{\dagger} \bar{\sigma}^{0}=\Psi^{\dagger} \sigma^{3}$ [24]. As shown in [24], (26) is expressed in explicit form as follows:

$$
\begin{aligned}
J^{\tau}= & \frac{1}{2 m}\left(\bar{\Psi} \bar{\sigma}^{\tau v}(t, r) \Psi\right)_{, v} \\
& -\frac{1}{2 m} \bar{\Psi}\left(\frac{i}{2} g^{\tau v} \overleftrightarrow{\partial_{v}}-e A^{\tau}\right) \Psi \\
& -\frac{i}{4 m} \bar{\Psi}\left[\bar{\sigma}^{v}(t, r), \bar{\sigma}_{, v}^{\tau}(t, r)\right] \Psi \\
& -\frac{i}{2 m} \bar{\Psi}\left[\bar{\sigma}^{v}(t, r) \Gamma_{v}, \bar{\sigma}^{\tau}(t, r)\right] \Psi \\
& -\frac{i}{4 m} \bar{\Psi}\left[\bar{\sigma}_{, v}^{v}(t, r), \bar{\sigma}^{\tau}(t, r)\right] \Psi
\end{aligned}
$$

The components of the Dirac current in the $2+1$ dimensional exponential expanding universe, $J^{0}$ and $J^{k}$, are

$$
\begin{aligned}
J^{0}= & \frac{1}{2 m} \partial_{k}\left[\bar{\Psi} \bar{\sigma}^{0 k}(t, r) \Psi\right]-\frac{1}{2 m} \bar{\Psi}\left(\frac{i}{2} \overleftrightarrow{\partial^{0}}-q A^{0}\right) \Psi \\
& -\frac{i}{2 m r} \exp (-H t) \bar{\Psi} \bar{\sigma}^{1} \bar{\sigma}^{0} \Psi
\end{aligned}
$$

and

$$
\begin{aligned}
J^{k}= & \frac{1}{2 m} \partial_{0}\left(\bar{\Psi} \bar{\sigma}^{0 k}(t, r) \Psi\right)+\frac{1}{2 m} \partial_{l}\left(\bar{\Psi} \bar{\sigma}^{l k}(t, r) \Psi\right) \\
& -\frac{1}{2 m} \bar{\Psi}\left(\frac{i}{2} \overleftrightarrow{\partial^{k}}-q A^{k}\right) \Psi
\end{aligned}
$$

$$
\begin{aligned}
& +i \frac{3 H}{2 m r} \exp (-H t) \bar{\Psi} \bar{\sigma}^{0} \bar{\sigma}^{k} \Psi \\
& +\delta_{k 2} i \frac{3 H}{2 m r} \exp (-H t) \bar{\Psi} \bar{\sigma}^{1} \bar{\sigma}^{2} \Psi
\end{aligned}
$$

where $k, l=1,2, \bar{\sigma}^{0 k}=i / 2\left[\bar{\sigma}^{0}, \bar{\sigma}^{k}(t, r)\right], \bar{\sigma}^{k l}=i / 2\left[\bar{\sigma}^{k}(t\right.$, $\left.r), \bar{\sigma}^{l}(t, r)\right]$, and $\delta_{k 2}$ is Dirac-delta function. Also these components can be rewritten in terms of the convective, the polarization, and magnetization parts as follows:

$$
J_{0}=\partial_{k} \mathbf{P}_{k}+\rho_{\text {convective }}-\frac{i}{2 m r} \exp (-H t) \bar{\Psi} \bar{\sigma}^{1} \bar{\sigma}^{0} \Psi
$$

and

$$
\begin{aligned}
J_{k}= & \partial_{0} \mathbf{P}_{k}+\partial_{l} \mathbf{M}_{[l k]}+J_{k \text { convective }} \\
& +i \frac{3 H}{2 m r} \exp (-H t) \bar{\Psi} \bar{\sigma}^{0} \bar{\sigma}^{k} \Psi \\
& +\delta_{k 2} i \frac{3 H}{2 m r} \exp (-H t) \bar{\Psi} \bar{\sigma}^{1} \bar{\sigma}^{2} \Psi
\end{aligned}
$$

where $\mathbf{P}_{0 k}$ are polarization densities and $\mathbf{M}_{[l k]}$ is magnetization density, and their explicit forms are given by

$$
P^{0 k}=\frac{1}{2 m} \bar{\Psi} \bar{\sigma}^{k 0}(t, r) \Psi
$$

and

$$
M^{[l k]}=\frac{1}{2 m} \bar{\Psi} \bar{\sigma}^{l k}(t, r) \Psi
$$

respectively. From these relations, the total polarizations, $p_{l}^{0}$, and magnetization, $\mu$, are defined as

$$
p_{l}^{0}=\int P^{0 k} d \Sigma_{k l}
$$

and

$$
\mu=\int M^{k l} d \Sigma_{k l}
$$

where $d \Sigma_{k l}$ is an hypersurface for $t=$ constant and $d \Sigma_{k l}=$ $\sqrt{|g|} d^{2} x=e^{2 H t} r d r d \phi[25]$.

Now, we are going to discuss the Dirac currents and the dipole moments expressions for the exponentially expending universe. So, inserting (17) and its conjugate into (26), we 
compute the components of the Dirac currents in asymptotic region as follows:

$$
\begin{aligned}
J^{0} & \approx \frac{H^{2}}{2 \lambda^{2} \pi^{2}} \frac{z^{2}}{r}, \\
J^{1} & \approx \frac{H^{3} z^{2}\left[(2 n+1)^{2}-z^{2}\right]}{4 \pi^{2} \lambda^{3}(2 n+1) r} \sin \left(\lambda r-\frac{k \pi}{2}\right), \\
J^{2} & \approx i \frac{H^{3} z^{2}\left[z^{2}-(2 n+1)^{2}\right]}{4 \pi^{2} \lambda^{3}(2 n+1) r} \sin \left(\lambda r-\frac{k \pi}{2}\right) .
\end{aligned}
$$

Similarly, substituting (17) and its conjugate in (32) and (33), the components of the polarization densities and the magnetization density are written as follows:

$$
\begin{aligned}
P^{1} & =A(z) J_{k+1 / 2}(\lambda r) J_{k-1 / 2}(\lambda r), \\
P^{2} & =B(z) \frac{1}{r} J_{k+1 / 2}(\lambda r) J_{k-1 / 2}(\lambda r), \\
M_{12} & =\frac{\lambda^{4} r}{8 \pi m^{3}}\left[J_{k+1 / 2}(\lambda r) J_{k+1 / 2}(\lambda r)\right. \\
& \left.-J_{k-1 / 2}(\lambda r) J_{k-1 / 2}(\lambda r)\right],
\end{aligned}
$$

respectively, where we use the following abbreviations:

$$
\begin{aligned}
& A(z)=i \frac{H^{3} z^{3}}{8 \pi \lambda^{2} m}\left[\frac{J_{n+1 / 2}(z)}{J_{n-1 / 2}(z)}-\frac{J_{n-1 / 2}(z)}{J_{n+1 / 2}(z)}\right], \\
& B(z)=-i A(z) .
\end{aligned}
$$

Giving to the polarization densities and magnetization density depending spacetime coordinates, we can say that particle production event takes place. To calculate the total polarizations and magnetization, we insert (37), the polarization densities, and magnetization density, into (34) and (35), respectively, and later integrate them on the hypersurface. Then, we obtain the total polarization densities (electric dipole moments) and magnetization density (magnetic dipole moment) as follows:

$$
\begin{aligned}
p^{1} & =0, \\
p^{2} & =\frac{\pi \lambda}{H^{2}} \frac{B(z)}{z^{2}}, \\
\mu & =0,
\end{aligned}
$$

where we use the integral representation of Bessel function [36] and $p^{1}$ and $p^{2}$ are total polarizations, i.e., electric dipole moment components, and also $\mu$ is total magnetization, i.e., magnetic dipole moment. From these results, we see that the particle creation events are affected from only $p^{2}$ total polarization density, electric dipole moment. On the other hand, in the limit $t \longrightarrow-\infty(z \longrightarrow \infty), p^{2}$ vanishes by the following way:

$$
p^{2} \longrightarrow \frac{z H}{4 \lambda m}\left[\frac{2 \cos ^{2}(z-n \pi / 2)-1}{\sin (2 z-n \pi)}\right] \rightarrow 0,
$$

that is, there is not particle production in this limit or the universe has a symmetric charge distribution in the beginning time and, of course, the universe has not any dipole moments, but, in the limit $t \longrightarrow+\infty(z \longrightarrow 0), p^{2}$ becomes

$$
\begin{aligned}
p^{2} & \longrightarrow \frac{e \hbar H}{4 \lambda m c^{2}}\left[\frac{z^{2}-\left(1-i\left(2 m c^{2} / \hbar H\right)\right)^{2}}{1-i\left(2 m c^{2} / \hbar H\right)}\right] \\
& \longrightarrow-\frac{e}{2 \lambda \delta} \exp (-i \delta),
\end{aligned}
$$

where $\hbar$ is Planck constant, $c$ is the speed of light, and $\delta=$ $2 m c^{2} / \hbar H$, and, thus, the universe has a permanent complex dipole moment, which it oscillates with Zitterbewegung frequency, $2 m c^{2} / \hbar$.

To calculate the Dirac current components for the Dirac particle in the $2+1$ dimensional exponentially expanding magnetized universe, we insert (24) and its conjugate in (26). Thus, we find that the current components are

$$
\begin{aligned}
J^{0} & \approx \frac{H^{2} k^{2}}{z^{2}\left(k^{2}-\kappa^{2}\right) \Gamma(2 \kappa)+e^{2} B_{0}^{2}\left(1-4 n^{2}\right) \Gamma(2 \kappa-2)} \\
& \cdot z^{3} \rho^{2 \kappa-1} e^{-\rho}[z \\
& \left.+\frac{\left(1-4 n^{2}\right)}{z\left(k^{2}-\kappa^{2}\right) \rho^{2}}[(2 k-\rho)(k+\kappa)+2 k \rho]^{2}\right] \\
J^{1} & \approx i \frac{4 n H^{3} e B_{0} k^{2} \kappa}{\pi\left(k^{2}-\kappa^{2}\right)} z^{4} \rho^{2 \kappa-2} e^{-\rho}[(2 k-\rho)(k+\kappa) \\
& +2 k \rho] \\
J^{2} & \approx \frac{4 H^{3} k^{3} z^{4}}{\pi\left(k^{2}-\kappa^{2}\right)} \\
& \cdot \frac{(\rho-2 k)(k+\kappa)+2 k \rho}{z^{2}\left(k^{2}-\kappa^{2}\right) \Gamma(2 \kappa)+\left(1-4 n^{2}\right) \Gamma(2 \kappa-2)} \\
& \cdot \rho^{2 \kappa-3} e^{-\rho} .
\end{aligned}
$$

Using (24) in (32) and in (33), the components of polarization and magnetization can be found as follows:

$$
\begin{aligned}
P^{1} & \approx \frac{2 H^{3} e B_{0} k^{2} z^{4} \kappa}{\left(k^{2}-\kappa^{2}\right) \pi m} \frac{(2 k-\rho)(k+\kappa)+2 k \rho}{2 z^{2} \lambda^{2} \kappa^{2} \Gamma(2 \kappa)+e^{2} B_{0}^{2}\left(1-4 n^{2}\right) \Gamma(2 \kappa-2)} \\
& \cdot \rho^{2 \kappa-2} e^{-\rho} \\
P^{2} & \approx i \frac{4 n H^{3} k^{3} z^{4}}{\pi m\left(k^{2}-\kappa^{2}\right)} \frac{[(2 k-\rho)(k+\kappa)+2 k \rho]}{z^{2}\left(k^{2}-\kappa^{2}\right) \Gamma(2 \kappa)+\left(1-4 n^{2}\right) \Gamma(2 \kappa-2)} \\
& \cdot \rho^{2 \kappa-3} e^{-\rho} \\
M_{12} & \approx \frac{2 H^{4} e B_{0} k^{3} z^{5}\left(4 n^{2}-1\right) \kappa}{\pi m\left(k^{2}-\kappa^{2}\right)\left[4 z^{2} \lambda^{2} \kappa^{2} \Gamma(2 \kappa)+e B_{0}\left(1-4 n^{2}\right) \Gamma(2 \kappa-2)\right]}
\end{aligned}
$$




$$
\begin{aligned}
& \cdot \rho^{2 \kappa-2} e^{-\rho} \\
& \times\left[\frac{z}{\left(1-4 n^{2}\right)}+\frac{1}{z\left(k^{2}-\kappa^{2}\right) \rho^{2}}[(2 k-\rho)(k+\kappa)+2 k \rho]^{2}\right]
\end{aligned}
$$

To calculate the total polarizations and total magnetization, we insert (43) into (34) and (35). Then, using the integral representation of Bessel function [36], we find the following dipole moments expressions:

$$
\begin{aligned}
p^{1} & \approx \frac{H e^{3} B_{0}^{3} k^{4} z^{2}}{\kappa^{3} m} \\
\cdot & \frac{2(k-\kappa)^{2} \Gamma(2 \kappa)}{z^{2}\left(k^{2}-\kappa^{2}\right) \Gamma(2 \kappa)+\left(1-4 n^{2}\right) \Gamma(2 \kappa-2)}, \\
p^{2} & \approx i \frac{2 n H e^{4} B_{0}^{4} k^{5} z^{2}}{\kappa^{4} m} \\
& \cdot \frac{\left(2 k^{2}-2 \kappa^{2}+4 k \kappa+\kappa-k\right) \Gamma(2 \kappa-1)}{z^{2}\left(k^{2}-\kappa^{2}\right) \Gamma(2 \kappa)+\left(1-4 n^{2}\right) \Gamma(2 \kappa-2)}, \\
\mu & \approx \frac{H^{2} e^{3} B_{0}^{3} k^{5}}{\left(k^{2}-\kappa^{2}\right) \kappa^{3} m} z^{3} .
\end{aligned}
$$

From these expressions, we see that, in finite time intervals, the particle creation is influenced by both polarization and magnetization components. On the other hand, in the limit $t \longrightarrow \infty(z \longrightarrow 0)$ the magnetic dipole moment, $\mu$, goes to zero faster than the electric dipole moment components, $p^{1}$ and $p^{2}$. Therefore, the electric dipole moments in the particle creation events become more dominant than the magnetic dipole moment in finite time intervals if there exists in an external constant magnetic field.

\section{Summary and Conclusion}

We exactly solve the Dirac equation in existence of the exponentially expanding magnetized and nonmagnetized universe and, from these solutions, derive some expressions for the Dirac current components and dipole moments. The particle creation in the exponentially expanding universe is only affected by the $p^{2}$ polarization in the finite time interval. However, this component goes to zero in the limit $t \longrightarrow-\infty$, i.e., in the beginning of the universe, but, in the limit $t \longrightarrow$ $+\infty$, the universe has a permanent complex dipole moment oscillating with Zitterbewegung frequency, $2 m c^{2} / \hbar: p^{2} \simeq$ $-(e / 2 \lambda \delta) \exp (-i \delta)$. The complexity of the dipole moment points out the conductivity of the exponentially expanding universe. Also, the universe has the electric and magnetic dipole moments which are dependent on time in existence of an external constant magnetic field with the expansion such that, in the limit $t \longrightarrow-\infty$, the dipole moment expressions become infinite, but, in the limit $t \longrightarrow \infty$, they go to zero. The dependence on time of the polarization and magnetization shows that the particle creation happens. Furthermore, in the limit $t \longrightarrow \infty(z \longrightarrow 0)$, the particle creation events are affected only via the polarization because the magnetization, $m$, goes to zero faster than $p^{1}$ and $p^{2}$. From the point of view, we point out that the exponential expansion of the universe causes a particle creation, a permanent complex electric dipole moment and asymmetric charge distribution, but, in existence of an external constant magnetic field with exponential expansion in time, the universe charge distribution is get and getting symmetric and thus all the dipole moments become zero as $t \longrightarrow \infty$.

\section{Data Availability}

No data were used to support this study.

\section{Conflicts of Interest}

The authors declare that there is no conflict of interests regarding the publication of this paper.

\section{References}

[1] L. Parker, "Particle creation in expanding universes," Physical Review Letters, vol. 21, no. 8, article no. 562, 1968.

[2] L. Parker, "Quantized fields and particle creation in expanding universes. I," Physical Review, vol. 183, no. 5, article no. 1057, 1969.

[3] L. Parker, "Quantized fields and particle creation in expanding universes. II," Physical Review D, vol. 3, no. 2, article no. 346, 1971.

[4] A. O. Barut and I. H. Duru, "Exact solutions of the Dirac equation in spatially flat Robertson-Walker space-times," Physical Review D, vol. 36, no. 12, article no. 3705, 1987.

[5] I. Sakalli and M. Halilsoy, "Solution of the Dirac equation in the near horizon geometry of an extreme Kerr black hole," Physical Review D: Particles, Fields, Gravitation and Cosmology, vol. 69, no. 12, article no. 124012, 2004.

[6] A. Zecca, "Comoving self-gravitating scalar field in the Newman Penrose formalism," International Journal of Theoretical Physics, vol. 45, no. 2, pp. 385-393, 2006.

[7] S. Moradi, "Exact solutions of dirac equation and particle creation in $(1+3)$-dimensional robertson-walker spacetime," International Journal of Theoretical Physics, vol. 48, no. 4, pp. 969-980, 2009.

[8] S. Moradi, "Particle creation in asymptotically Minkowskian spacetimes," Journal of Geometry and Physics, vol. 59, no. 2, pp. 173-184, 2009.

[9] N. G. Sarkar and S. Biswas, "Particle production in curved spacetime," Pramana, vol. 50, no. 2, pp. 109-131, 1998.

[10] N. G. Sarkar and S. Biswas, "Particle production in de Sitter space-time," International Journal of Modern Physics A, vol. 15, no. 4, pp. 497-508, 2000.

[11] Y. Sucu and N. Unal, "Vector bosons in the expanding universe," European Physical Journal C, vol. 44, no. 2, pp. 287-291, 2005.

[12] Y. Sucu and N. Ünal, "Dirac equation in Euclidean NewmanPenrose formalism with applications to instanton metrics," Classical and Quantum Gravity, vol. 21, no. 6, pp. 1443-1451, 2004.

[13] M. Dernek, S. Gurtas Dogan, Y. Sucu, N. Unal, and J. Phys, "Relativistic quantum mechanical spin-1 wave equation in $2+$ 1 dimensional spacetime," Turkish Journal of Physics, vol. 42, pp. 509-526, 2018. 
[14] C. Tekincay, Y. Sucu, and J. Phys, "Photon in a cylindrical resonant cavity," Turkish Journal of Physics, vol. 42, pp. 175-182, 2018.

[15] H. Hassanabadi, E. Maghsoodi, and S. Zarrinkamar, "Dirac equation with vector and scalar cornell potentials and an external magnetic field," Annals of Physics, vol. 525, no. 12, pp. 944-950, 2013.

[16] H. Hassanabadi, H. Sobhani, and W. S. Chung, "Scattering study of fermions due to double Dirac delta potential in quaternionic relativistic quantum mechanics," Advances in High Energy Physics, vol. 2018, Article ID 8124073, 7 pages, 2018.

[17] M. Hosseinpour, H. Hassanabadi, and M. de Montigny, "The Dirac oscillator in a spinning cosmic string spacetime," The European Physical Journal C, vol. 79, article 311, 2019.

[18] A. N. Ikot, H. Hassanabadi, N. Salehl, H. P. Obong, and M. C. Onyeaju, "Scattering states of Cusp potential in minimal length Dirac equation," Indian Journal of Physics, vol. 89, no. 11, pp. 1221-1226, 2015.

[19] M. Hamzavi, A. A. Rajabi, and H. Hassanabadi, "Exactly complete solutions of the Dirac equation with pseudoharmonic potential including linear plus Coulomb-like tensor potential," International Journal of Modern Physics A, vol. 26, no. 7-8, pp. 1363-1374, 2011.

[20] S. Ghosh, "Creation of spin 1/2 particles and renormalization in FLRW spacetime," Physical Review D: Particles, Fields, Gravitation and Cosmology, vol. 91, no. 12, article no. 124075, 2015.

[21] S. Bhattacharjee, S. Saha, and S. Chakraborty, "Does particle creation mechanism favour formation of black hole or naked singularity?" The European Physical Journal C, vol. 78, article 488, 2018.

[22] A. Jawad, S. Sami, and S. Rafique, "Thermodynamics of various entropies in specific modified gravity with particle creation," Advances in High Energy Physics, vol. 2018, Article ID 9471346, 17 pages, 2018.

[23] A. Bhoonah, "Gravitational fermion creation during an anisotropic phase of cosmological expansion," Physical Review D: Particles, Fields, Gravitation and Cosmology, vol. 99, article 105003, Article ID 105003, 2019.

[24] Y. Sucu and N. Ünal, "Exact solution of Dirac equation in 2+1 dimensional gravity," Journal of Mathematical Physics, vol. 48, no. 5, article no. 052503, 2007.

[25] S. Gurtas Dogan, G. Gecim, and Y. Sucu, "A new approach to compute the dipole moments of a Dirac electron," 2019, https://arxiv.org/abs/1907.02393.

[26] O. Gurtug, S. Mazharimousavi, and M. HHalilsoy, "Classical and quantum analysis of an Einstein-Scalar solution in $2+1$ dimensions," The European Physical Journal plus, vol. 132, no. 4, article no. 161, 2017.

[27] O. Gurtug, M. Halilsoy, and S. Habib Mazharimousavi, "Quantum probes of timelike naked singularities in $2+1$-dimensional power-law spacetimes," Advances in High Energy Physics, vol. 2015, Article ID 684731, 10 pages, 2015.

[28] G. Gecim, Y. Kucukakca, and Y. Sucu, "Noether gauge symmetry of dirac field in $(2+1)$-dimensional gravity," Advances in High Energy Physics, vol. 2015, Article ID 567395, 7 pages, 2015.

[29] G. Gecim and Y. Sucu, "Dirac field as a source of the inflation in $2+1$ dimensional teleparallel gravity," Advances in High Energy Physics, vol. 2017, Article ID 2056131, 9 pages, 2017.

[30] G. Gecim and Y. Sucu, "Cosmological Solutions in $2+1-$ Dimensional New Massive Gravity in the Presence of the DIRac Field," Gravitation \& Cosmology, vol. 25, no. 2, pp. 179-183, 2019.
[31] G. Gecim and Y. Sucu, "Quantum gravity effect on the tunneling particles from Warped-AdS 3 black hole," Modern Physics Letters $A$, vol. 33, no. 28, article no. $1850164,2018$.

[32] G. Gecim and Y. Sucu, "The GUP effect on Hawking radiation of the $2+1$ dimensional black hole," Physics Letters B, vol. 773, pp. 391-394, 2017.

[33] G. Gecim, Y. Sucu, and Adv., "Quantum gravity effect on the tunneling particles from $2+1$-dimensional new-type black hole," Advances in High Energy Physics, vol. 2018, Article ID 8728564, 7 pages, 2018.

[34] S. Weinberg, Gravitation and Cosmology: Principles and Applications of the General Theory of Relativity, John Wiley and Sons, New York, NY, USA, 1972.

[35] M. Kenmoku, S. Uchida, and T. Matsuyama, "Conical singular solutions in $(2+1)$-dimensional gravity employing the ADM canonical formalism," International Journal of Modern Physics $D$, vol. 12, no. 4, pp. 677-687, 2003.

[36] I. S. Gradshteyn and I. M. Ryzhik, Table of Integrals, Series, and Products, Elsevier Press, New York, NY, USA, 7th edition, 2007.

[37] J. D. Jackson, Classical Electrodynamics, John Wiley and Sons, New York, NY, USA, 1999. 

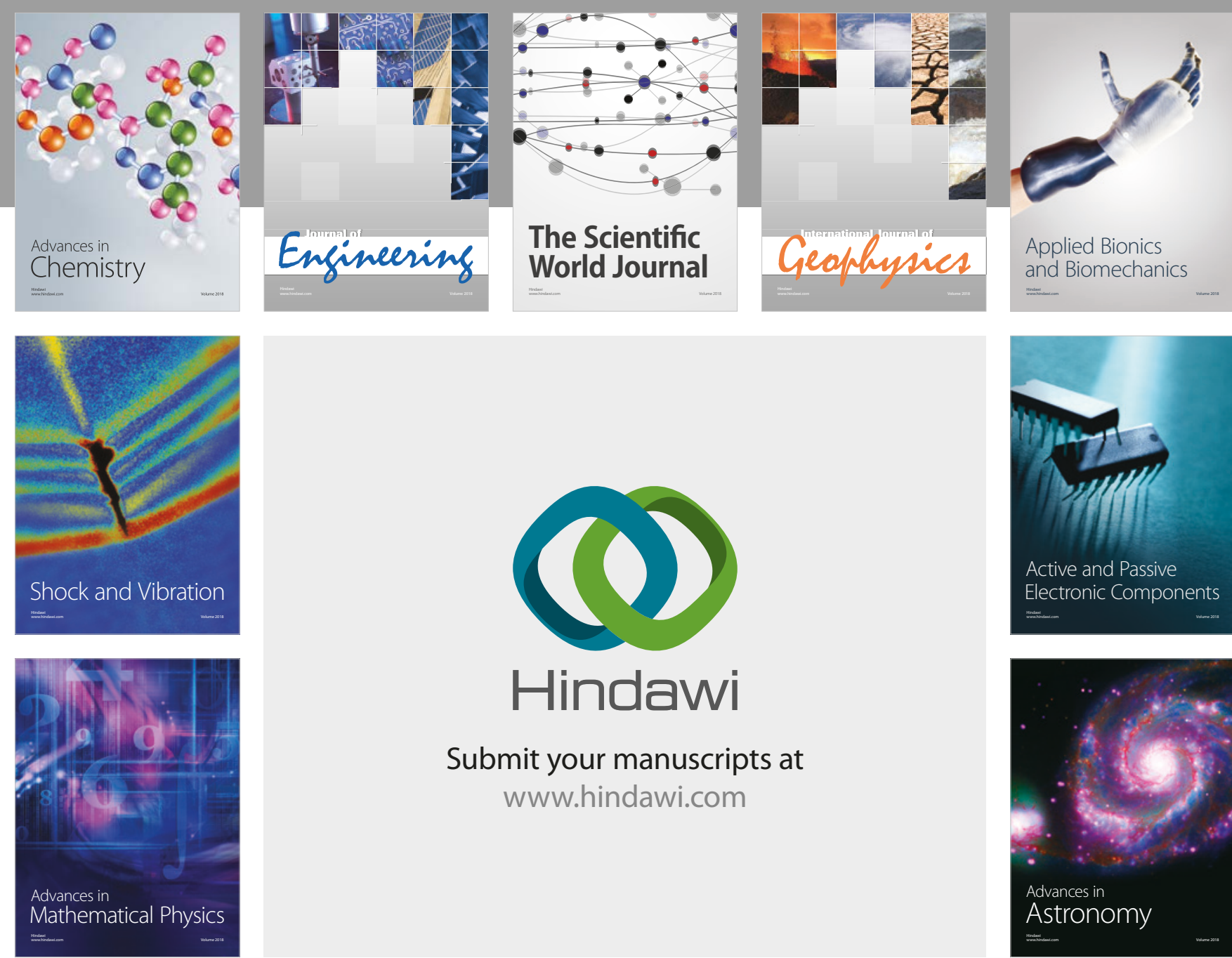

Submit your manuscripts at

www.hindawi.com

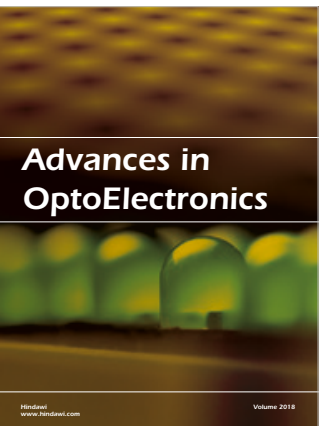

\section{Rotcting Machinery}
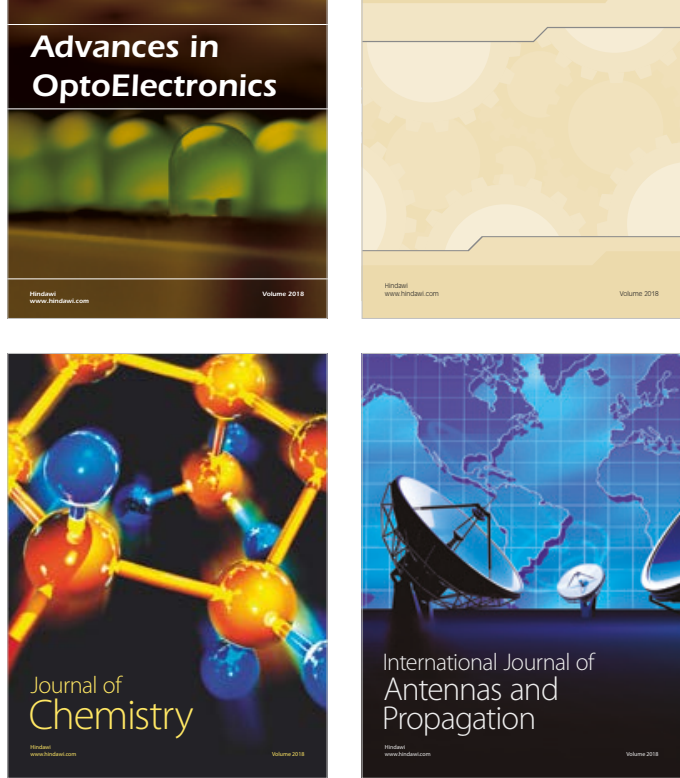

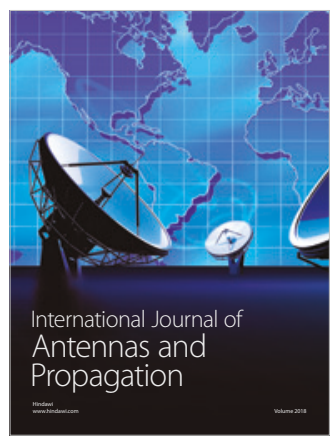

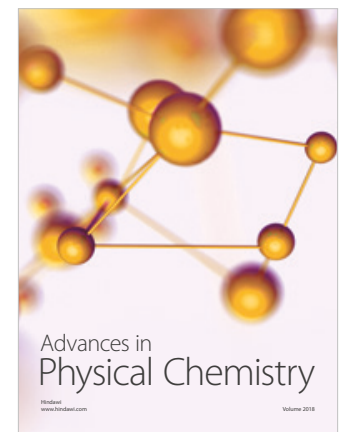

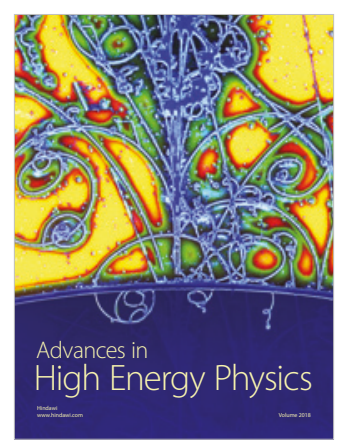

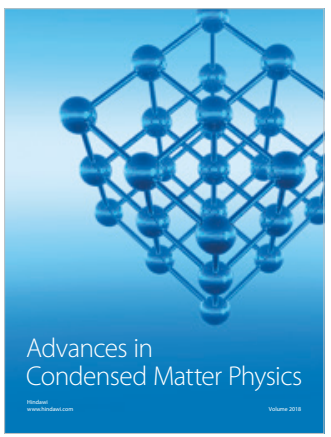

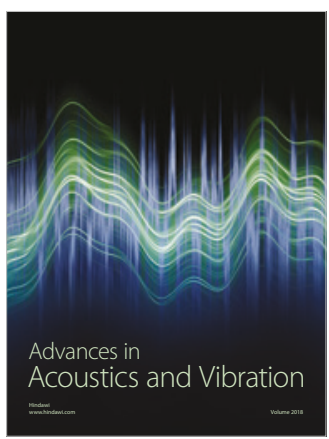

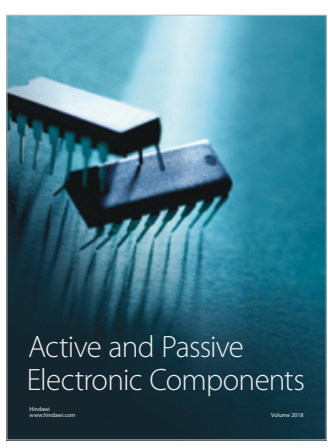
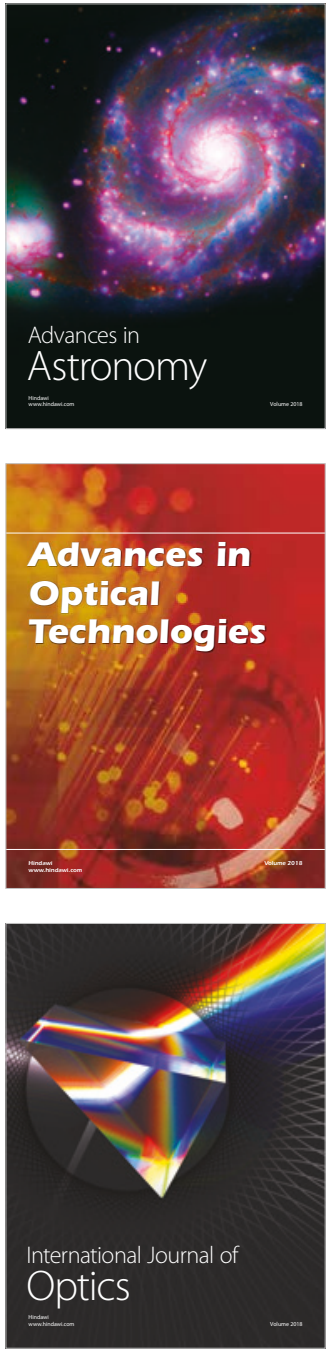